\title{
Stabilized Space-time finite element approximations for a class of quasilinear parabolic problems
}

\author{
Ioannis Toulopoulos* \\ * Institute of Computational Mathematics Johannes Kepler University Linz, Austria \\ e-mail:ioantlp@gmail.com
}

\begin{abstract}
In this talk, we present continuous space-time finite element methods for solving quasilinear parabolic problems of the type $u_{t}-\operatorname{div} A(\nabla u)=f$, in $\bar{Q}_{T}=\bar{\Omega} \times[0, T]$, in space and time simultaneously. We focus mainly on the problems with $A(\mathbf{a})=(\varepsilon+|\mathbf{a}|)^{p-2} \mathbf{a}$ with parameters $\varepsilon>0$ and $p>1$. In our approach we consider the time variable $t$ as just another variable, say, $x_{d+1}$ if $x_{1}, \ldots, x_{d}$ are the spatial variables, and the time derivative as a strong convection in the direction $x_{d+1}$. Multiplying the associated PDE by a test function depending on spatial and time variable and then applying integration by parts we lead to the space-time variational formulation. Based on this we develop the finite element approach. We treat numerically the discretization of the time derivative in a stable way by using Streamline-Upwind Petrov-Galerkin (SUPG) techniques. Using the results presented in [1] and [2], we derive discretization error estimates taking into account that the exact solution can exhibit some anisotropic regularity behavior, i.e., different regularity properties with respect to the time and to the space directions. The impact of this in the discretization error analysis is the use of different $L^{p}$ norms for the error variations in time and in space correspondingly. In the last part of the talk, we present numerical results which confirm the theoretical convergence rate estimates. This talk is based on [3]. This work is supported by the project JKU-LIT-2017-4-SEE-004, at the Institute of Computational Mathematics Johannes Kepler University Linz.
\end{abstract}

\section{REFERENCES}

[1] L. Diening, M. Růžička. Interpolation operators in Orlicz-Sobolev spaces. Numer. Math. 107, (2007) 107-129.

[2] S. Nakov and I. Toulopoulos. Convergence estimates of finite elements for a class of quasilinear elliptic problems. under review 2020,

[3] I. Toulopoulos. Space-time finite element approximations for quasilinear parabolic problems, under review 2021. 